\title{
Armonización entre la gestión documental, la calidad y la seguridad de la información en una institución de educación superior*
}

\author{
Jorge William Triana Torres ${ }^{* *}$ \\ Ingrid Carolina Moreno Rodríguez ${ }^{* * *}$
}

Recibido: 10 de agosto de 2020

Revisado: 27 de enero de 2021

Aceptado: 14 de agosto de 2021

Citar como:

Triana Torres, J. W. y Moreno Rodríguez, I. C. (2021). Armonización entre la gestión documental, la calidad y la seguridad de la información en una institución de educación superior. Signos, Investigación en Sistemas de Gestión, 13(2). https://doi.org/10.15332/24631140.6664

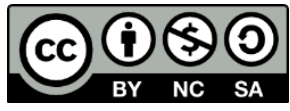

\footnotetext{
* Artículo resultado de investigación.

** Magíster en Calidad y Gestión Integral por la Universidad Santo Tomás, especialista en Administración y Gerencia de Sistemas de la Calidad, bibliotecólogo y archivista. Universidad Santo Tomás. Bogotá, Colombia. Correo electrónico: jorgetriana@usantotomas.edu.co, jwilliam38@gmail.com; ORCID: https://orcid.org/0000-0002-3207-6451; CvLAC: https://scienti.minciencias.gov.co/cvlac/visualizador/generarCurriculoCv.do?cod rh $=0001850124$ ${ }^{* * * *}$ Magíster en Gestión Documental y Administración de Archivos por la Universidad de La Salle, especialista en Administración y Gerencia de Sistemas de la Calidad, y administradora de empresas. Grupo de Investigación GEAMEC, Universidad Santo Tomás. Bogotá, Colombia. Correo electrónico: ingridcmoreno@usantotomas.edu.co; cmrcalidad21@gmail.com; ORCID: https://orcid.org/0000-0003-3624-0877; CvLAC:

http://scienti.colciencias.gov.co:8081/cvlac/visualizador/generarCurriculoCv.do?cod rh $=0000101$ $\underline{575}$
}

Signos, Investigación en Sistemas de Gestión

ISSN: 2145-1389 | e-ISSN: 2463-1140 | DOI: https://doi.org/10.15332/24631140

Vol. 13 N.0 2 | julio-diciembre de 2021 


\section{Resumen}

La gestión documental es un proceso transversal encaminado a facilitar el uso de la información en las organizaciones para cumplir su objeto misional y preservar el patrimonio documental. El tratamiento adecuado de la información se convierte en uno de los valores intangibles y sustanciales para gestionar la organización como un todo. Este se trata de articular los sistemas de gestión para asegurar la eficacia en el logro de los objetivos. El presente artículo expone los resultados de una investigación descriptiva con enfoque mixto, orientada al desarrollo de una metodología de armonización entre los sistemas de gestión documental, calidad y seguridad de la información en una institución de educación superior. En la primera fase se aplicó un diagnóstico para verificar el nivel de cumplimiento de los sistemas mencionados, en la segunda fase se identificaron los puntos de inflexión, en la tercera fase se diseñó la metodología de armonización y en la cuarta fase se realizó la validación de la metodología propuesta. Entre los principales resultados se destaca la identificación de los puntos de inflexión y una guía metodológica para orientar la armonización. Se concluye que, a pesar de existir algunas diferencias de contenido y procesos de los sistemas estudiados, predominan las semejanzas.

Palabras clave: planificación de los archivos, sistema de información integrada, conservación de documentos, cultura del trabajo, protección de datos.

\section{Harmonization between document management, quality and information security in a Higher Education Institution}

\section{Abstract}

Document management is a cross-cutting process aimed at facilitating the use of information in organizations to fulfill their mission purpose and preserve the documentary heritage. The proper treatment of information becomes one of the intangible and substantial values to 
manage the organization as a whole. This is about articulating the management systems to ensure efficiency in objectives acomplishment. This article presents the results of a mixed approach descriptive research, oriented to the development of a harmonization methodology between document management, quality and information security systems in a higher education institution. In the first phase a diagnosis was applied to verify the compliance level with the aforementioned systems; in the second phase the turning points were identified, in the third phase the harmonization methodology was designed and in the fourth phase the proposed methodology was validated. Among the main results, the identification of the turning points and a methodological guide to guide harmonization stand out. It is concluded that, despite some differences in content and processes of the systems studied, similarities predominate.

Keywords: archives planning, integrated information system, document preservation, work culture, data protection.

\section{Harmonização entre gestão documental, qualidade e segurança da informação em uma instituição de ensino superior}

\section{Resumo}

O gestao documental é um processo transversal que visa facilitar o uso da informação nas organizações para cumprir sua missão e preservar o patrimônio documental. O tratamento adequado das informações tornase um dos valores intangíveis e substanciais para administrar a organização como um todo. Trata-se de articular os sistemas de gestão para garantir a eficácia na realização dos objetivos. Este artigo apresenta os resultados de uma pesquisa descritiva com uma abordagem mista, que visa o desenvolvimento de uma metodologia de harmonização entre os sistemas de gestão documental, qualidade e segurança da informação em uma instituição de ensino superior. Na primeira fase, foi aplicado um diagnóstico para verificar o nível de conformidade dos sistemas 
supramencionados; na segunda fase, foram identificados os pontos de viragem; na terceira fase, foi projetada a metodologia de harmonização; e na quarta fase, foi validada a metodologia proposta. Entre os principais resultados, destacam-se a identificação dos pontos de viragem e um guia metodológico para orientar a harmonização. Conclui-se que predominam as semelhanças apesar de algumas diferenças no conteúdo e nos processos dos sistemas analizados.

Palavras-chave: planejamento de arquivos, sistema de informação integrado, retenção de dados, cultura de trabalho, proteção de dados.

\section{Introducción}

Actualmente, en Colombia se ha posicionado la gestión documental como un factor clave que facilita la toma de decisiones y da valor agregado al uso de la información de una organización, para el cumplimiento de su objeto misional y su posicionamiento en entornos económicos y sociales cada vez más complejos, competitivos y cambiantes. Estos aspectos obligan a las instituciones a cumplir no solo con las disposiciones reglamentarias, sino a adoptar modelos de buenas prácticas que les permitan armonizar integralmente sus sistemas de gestión.

El Estado colombiano ha promulgado legislación relacionada con la política y lineamientos de gestión documental, a su vez el Instituto Colombiano de Normas Técnicas (Icontec), como instancia de normalización en Colombia, ha adaptado y homologado normas técnicas colombianas como referentes para el cumplimiento de requisitos, procesos y actividades de la gestión de la información organizacional.

Se destaca como un antecedente relacionado la investigación adelantada por Puentes (2016), a partir de la cual se presenta una "Propuesta metodológica para articular la gestión documental con los requisitos de la Ley General de Archivos y la Norma Técnica Internacional ISO 9001:2015” (p.82). Esta facilitó el planteamiento de un instrumento de cohesión a 
utilizar entre la gestión de la calidad y la gestión documental, para la apropiada planeación, ejecución de procesos de la organización y conservación de los archivos físicos y electrónicos.

Cabe resaltar, además, los resultados de la investigación de Moreno (2018), pues permitío identificar la integración y el trabajo colaborativo que puede existir entre estos dos sistemas a través de los cuales se gestiona información en una institución de educación superior (IES), para optimizar recursos y consolidar planes de mejoramiento desde su estructura al asociar los procesos y que, desde la generación de la información hasta su disposición final, sea coherente la articulación de los sistemas abordados.

Acorde con Bustelo (2012), los sistemas de gestión deben identificar la creación y el control de documentos como un proceso de soporte que permite la evaluación y auditoría de los sistemas, por un lado, la documentación propia del sistema de gestión, así como la información generada por los procesos de negocio. Esta condición tiene como ventaja que se pueda ofrecer más confianza, funcionalidad y amigabilidad del sistema y, por lo mismo, implica que la organización deberá contar con un sistema de gestión documental, que actúe como moderador de todos los otros sistemas de gestión (p. 16).

Esta teoría es corroborada por Peña (2009), quien afirma que todos los sistemas de gestión, especialmente aquellos que han sido normalizados por medio de consensos nacionales o internacionales, tienen puntos de inflexión, que resultan compatibles entre sí, y también elementos complementarios, específicos de su campo. Esto implica efectuar un análisis previo en aspectos como la relación con la planificación estratégica, metas, estructura organizacional, recursos, operacionalización, evaluación de resultados, acciones de mejora, entre otros. 
La investigación se desarrolló en una IES con sede en Bogotá, la cual tiene una amplia trayectoria de más de 30 años y gran reconocimiento por su acreditación institucional a nivel nacional e internacional, cuenta con un sistema de gestión de la calidad y la certificación en ISO-9001-2015 otorgada por el Icontec. Actualmente, se encuentra en proceso de desarrollo del sistema de gestión integrado, conformado por la calidad, la gestión ambiental y seguridad y la salud en el trabajo. En términos de la gestión documental, se podría afirmar que en la institución existe un importante desarrollo normativo frente a la legislación y estándares de buenas prácticas, sin embargo, hay un gran vacío sobre cómo deben articularse entre sí. Por esta razón surge la situación problemática en la que se fundamenta la investigación, formulada a partir de la siguiente pregunta: ¿̇cómo se pueden armonizar los sistemas de gestión documental, calidad y seguridad de la información, en una IES, para dar cumplimiento a los requisitos de la legislación colombiana y las normas técnicas colombianas NTC-ISO 30301, 27001 y 9001, en pro de la adecuada administración, uso, manejo, seguridad, disposición y preservación de su información institucional?

A partir de la definición del problema, se planteó como objetivo principal elaborar una propuesta metodológica para la armonización del sistema de gestión documental con los sistemas de gestión de calidad y seguridad de la información, al atender la legislación y las normas técnicas colombianas NTC-ISO 30301, 27001 y 9001, con el propósito de facilitar la disponibilidad, uso y preservación de la información en una IES. Dicho objetivo se desarrolló a través de 3 objetivos específicos que consistieron en la realización de un diagnóstico en la institución objeto de estudio, el análisis de las correlaciones existentes entre el sistema de gestión documental y los sistemas de gestión de calidad y seguridad de la información, la elaboración de una metodología de armonización de los 
sistemas de gestión conforme con los criterios legales y normativos vigentes, y, finalmente, la validación a través de la consulta a expertos.

\section{Metodología}

De acuerdo con las características descritas en los objetivos, los antecedentes y el marco referencial, se aplicó una investigación descriptiva con enfoque mixto, la cual se desarrolló a través de 4 fases.

La fase de diagnóstico se llevó a cabo a partir de una herramienta diseñada por una matriz de Excel, que refleja el estado actual del nivel de cumplimiento o grado de implementación de los procesos de gestión documental en la institución, establecidos en el Artículo 2.8.2.5.9 del Decreto 1080 de 2015, las actividades de los procesos de gestión documental, señalados en la guía para la implementación de un programa de gestión documental del Archivo General de la Nación (AGN), las actividades generales de los procesos y controles descritos en las NTC-ISO 30301 y 27001, así como los requisitos generales de información documentada de la NTC-ISO 9001.

La fase de análisis se realizó a partir de las variables cualitativas y facilitó la identificación de los puntos de inflexión existentes entre el sistema de gestión documental y los sistemas de gestión de calidad y seguridad de la información. También se incluyeron los requisitos generales contemplados en las normas técnicas colombianas NTC-ISO 30301, 27001 y 9001.

La fase de diseño estuvo orientada a la construcción de una guía para la armonización de los procesos de gestión documental con los sistemas de gestión de calidad y seguridad de la información, a partir de la cual se identificaron los puntos de inflexión y se unificaron los criterios básicos para lograr la articulación entre estos sistemas, de tal forma que pueda ser adoptada por una IES. 
La fase de validación correspondió al proceso de verificación de los contenidos de la guía utilizando el Coeficiente de concordancia W de Kendall (Escobar-Pérez y Cuervo-Martínez, 2008, p. 32) con el fin de conocer el grado de acuerdo entre los expertos, en términos de claridad, pertinencia y su aplicabilidad. A través de una técnica de muestreo básico para métodos mixtos (Hernández, 2014), se determinó una población de 20 personas con perfil profesional entre las áreas de TIC, gestión de la calidad, auditoría interna y gestión documental de la IES; al definir una muestra de 7 personas, a quienes se les aplicó el instrumento para dicha validación, entre ellos, a los directores y profesionales de las áreas referidas, por ser las instancias directamente relacionadas con los temas objeto de la investigación.

Teniendo en cuenta el diseño explicativo secuencial (DEXPLIS) (Hernández, 2014, p. 554), se definieron las variables cuantitativas correspondientes a los valores porcentuales obtenidos en la aplicación del diagnóstico y que dieron como resultado el nivel de cumplimiento de las actividades de los procesos del Decreto 1080 del 2015 y los requisitos y actividades de las NTC-ISO 30301, 9001 y 27001 (ver figura 1). 
Figura 1. Esquema de variables cuantitativas y cualitativas
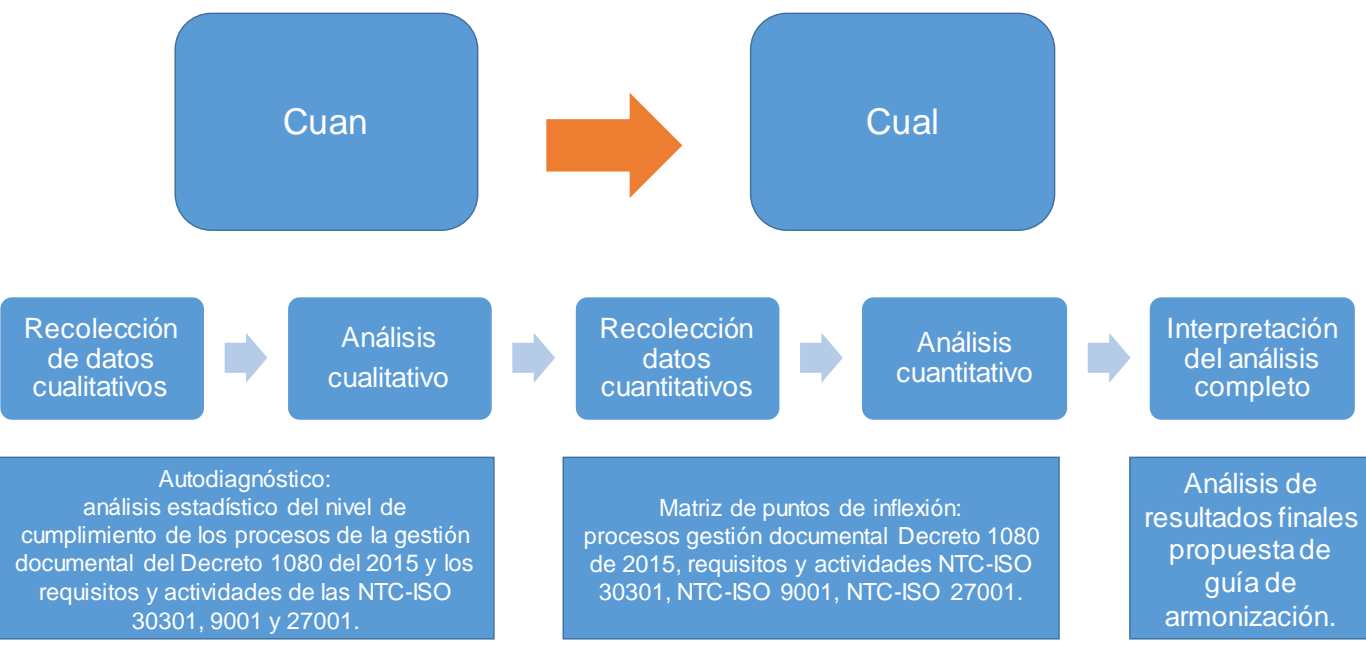

Análisis de

Matriz de puntos de inflexión:

procesos gestión documental Decreto 1080

de 2015, requisitos y actividades NTC-ISO

30301, NTC-ISO 9001, NTC-ISO 27001.

resultados finales

propuesta de

guía de

armonización.

Fuente: elaboración propia (2019) basada en Hernández (2014, p. 554).

Las categorías, subcategorías y criterios estuvieron ligados con las

preguntas de la investigación y los objetivos específicos propuestos, identificando las siguientes (ver tabla 1):

Tabla 1. Categorías y subcategorías

\begin{tabular}{c|c|}
\hline Categorías & Subcategorías - Criterios \\
\hline Autodiagnóstico & Recolección de información \\
& Análisis de datos \\
\hline & Interpretación de los resultados \\
\hline
\end{tabular}

Procesos de gestión documental, artículo. 2.8.2.5.9 de Decreto 1080 de 2015.

Actividades de los procesos de gestión documental: guía para la implementación de un programa de gestión documental.

Matriz de puntos de inflexión

Actividades generales de los procesos del anexo A: procesos y controles de la NTC-ISO 30301.

Actividades generales de los subobjetivos del anexo A: objetivos de control y controles de referencia de la NTC-ISO 27001.

Signos, Investigación en Sistemas de Gestión

ISSN: 2145-1389 | e-ISSN: 2463-1140 | DOI: https://doi.org/10.15332/24631140

Vol. 13 N.0 2 | julio-diciembre de 2021 


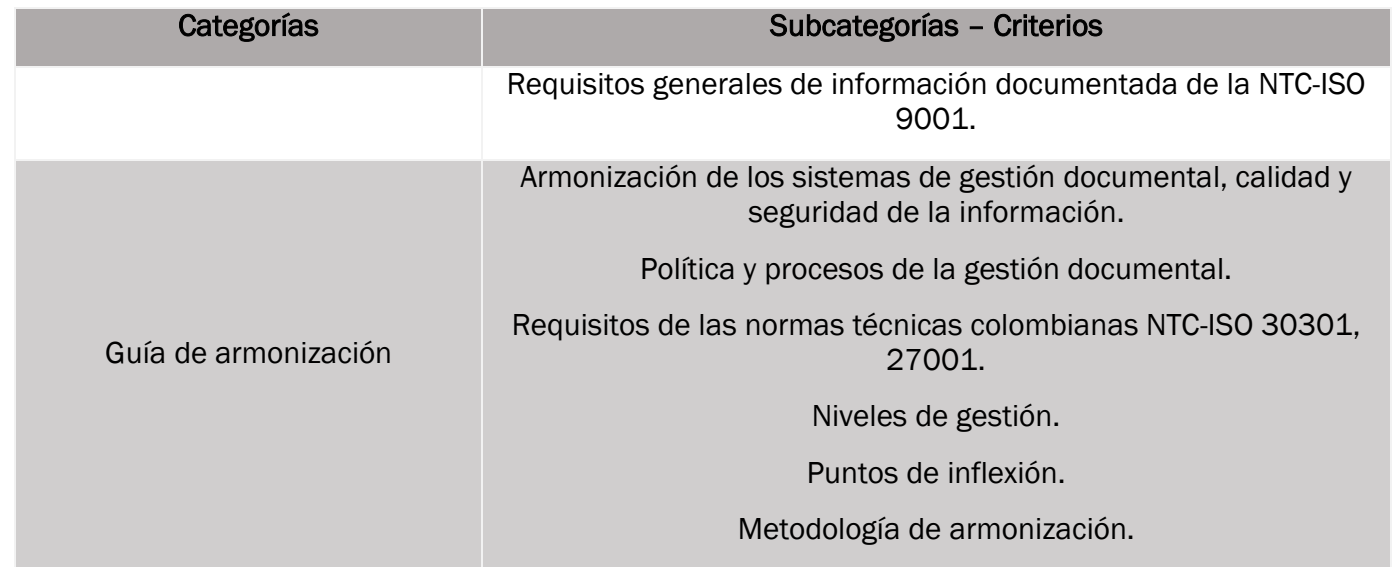

Elaboración del cuestionario de validación.

Definición de la metodología de validación.

Selección de expertos.

Consulta de expertos

Entrega de la guía y cuestionario a expertos.

Recepción de cuestionarios.

Análisis de resultados.

Calcular la concordancia entre expertos.

Elaboración de conclusiones,

Fuente: elaboración propia (2019).

\section{Para facilitar la recolección de la información y poder analizar las} variables, categorías, subcategorías y criterios de la investigación, se utilizaron los siguientes recursos, descritos en la tabla 2.

Tabla 2. Instrumentos y técnicas de investigación

\begin{tabular}{c|c} 
Objetivos específicos & Instrumentos y técnicas de investigación \\
$\begin{array}{c}\text { Realizar un diagnóstico de la situación actual de } \\
\text { los sistemas de gestión documental y sistemas } \\
\text { de gestión de calidad y seguridad de la }\end{array}$ & Revisión bibliográfica. \\
información, con el fin de verificar el grado de \\
$\begin{array}{c}\text { cumplimiento de la legislación archivística y los } \\
\text { requisitos establecidos en las normas técnicas } \\
\text { colombianas NTC-ISo 30301, } 27001 \text { y } 9001 .\end{array}$ & Observación directa. \\
\hline $\begin{array}{c}\text { Identificar los puntos de inflexión, tales como los } \\
\text { procesos y requisitos del sistema de gestión } \\
\text { documental y los sistemas de gestión de calidad } \\
\text { y seguridad de la información, con el propósito } \\
\text { de determinar las convergencias entre la } \\
\text { normatividad y los sistemas de gestión } \\
\text { estudiados. }\end{array}$ & Autodiagnóstico. \\
\hline
\end{tabular}

Signos, Investigación en Sistemas de Gestión

ISSN: 2145-1389 | e-ISSN: 2463-1140 | DOI: https://doi.org/10.15332/24631140

Vol. 13 N.0 2 | julio-diciembre de 2021 


\begin{tabular}{|c|c|}
\hline Objetivos específicos & Instrumentos y técnicas de investigación \\
\hline $\begin{array}{l}\text { Diseñar una guía de armonización de los } \\
\text { procesos de gestión documental con los } \\
\text { sistemas de gestión de calidad y seguridad de la } \\
\text { información, atendiendo lo dispuesto en la } \\
\text { legislación archivística y las normas técnicas } \\
\text { colombianas NTC-ISO 30301, } 27001 \text { y } 9001 .\end{array}$ & $\begin{array}{c}\text { Revisión bibliográfica. } \\
\text { Análisis de contenido. } \\
\text { Documento guía. }\end{array}$ \\
\hline $\begin{array}{l}\text { Validar la guía de armonización de los procesos } \\
\text { de gestión documental con los sistemas de } \\
\text { gestión de calidad y seguridad de la información, } \\
\text { mediante la consulta de expertos, con el } \\
\text { propósito de confirmar su claridad, pertinencia y } \\
\text { aplicabilidad. }\end{array}$ & $\begin{array}{l}\text { Revisión bibliográfica. } \\
\text { Cuestionario. } \\
\text { Herramienta metodológica. } \\
\text { Consulta de expertos. }\end{array}$ \\
\hline \multicolumn{2}{|l|}{ Fuente: elaboración propia (2019). } \\
\hline $\begin{array}{l}\text { Durante el desarrollo de la investigac } \\
\text { directores y profesionales de soporte } \\
\text { calidad, gestión documental y de la di } \\
\text { interna de la IES. En síntesis, se pudo } \\
\text { cumplimiento de las actividades de lo } \\
\text { acorde con el Decreto } 1080 \text { de } 2015, \\
\text { actividades exigen un esfuerzo presur } \\
\text { incorporación de herramientas, proce } \\
\text { tecnológicas para la gestión documen } \\
\text { muchas organizaciones. En la figura } \\
\text { cumplimiento. }\end{array}$ & $\begin{array}{l}\text { se contó con el apoyo de los } \\
\text { las áreas de TIC, gestión de } \\
\text { tora de la oficina de auditoría } \\
\text { ferir que existe un alto grado de } \\
\text { rocesos de la gestión documental } \\
\text { endo en cuenta que muchas de las } \\
\text { stal de las instituciones y la } \\
\text { nientos y aplicaciones } \\
\text { que aún son incipientes en } \\
\text { presenta este grado de }\end{array}$ \\
\hline
\end{tabular}

Signos, Investigación en Sistemas de Gestión

ISSN: 2145-1389 | e-ISSN: 2463-1140 | DOI: https://doi.org/10.15332/24631140

Vol. 13 N.० 2 | julio-diciembre de 2021 
Figura 2. Grado de cumplimiento de procesos de gestión documental

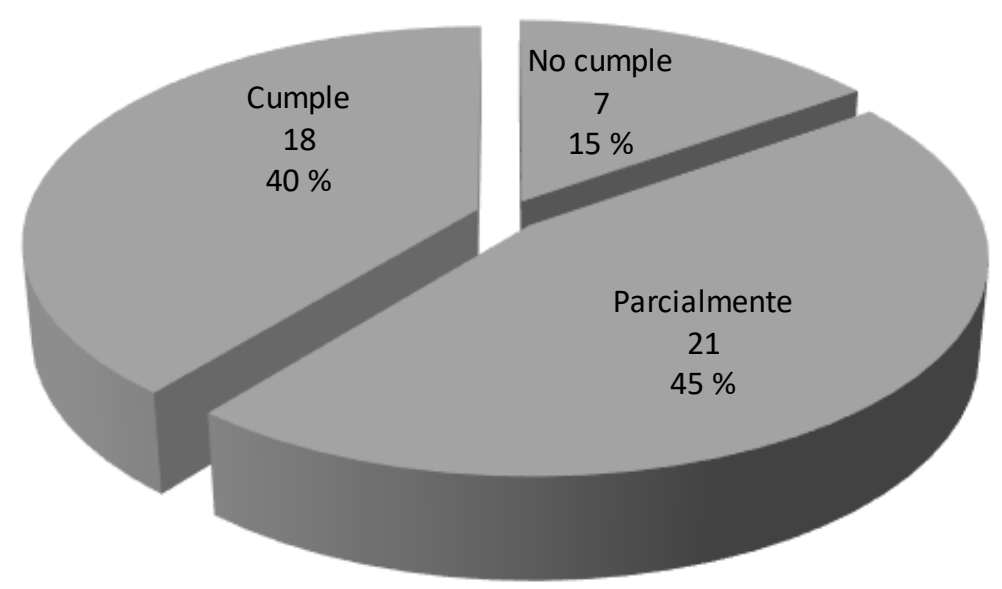

Fuente: elaboración propia (2019).

En términos generales, existe un alto cumplimiento de los procesos, actividades y requisitos planteados en la legislación archivística y las normas técnicas de gestión de calidad y seguridad de la información, sobre todo en lo atinente al compromiso de la alta dirección, formulación de políticas, toma de conciencia frente a la importancia de los sistemas, cumplimiento de requisitos e identificación de responsabilidades. Así mismo, las oportunidades de mejora en cada norma confluyen en los mismos aspectos, es decir que, para lograr la totalidad del cumplimiento, se requiere fortalecer la adquisición, el desarrollo, uso y apropiación de herramientas, procedimientos y aplicaciones tecnológicas para la adecuada gestión de la calidad, documental y de seguridad de la información.

Con la aplicación de la matriz de puntos de inflexión se logró la identificación de las correlaciones existentes entre los procesos de la 
gestión documental y los aspectos y requisitos de los sistemas de gestión de la calidad y seguridad de la información, en la tabla 3 se identifican dichos puntos frente a los sistemas de gestión mencionados.

Signos, Investigación en Sistemas de Gestión

ISSN: 2145-1389 | e-ISSN: 2463-1140 | DOI: https://doi.org/10.15332/24631140

Vol. 13 N.o 2 | julio-diciembre de 2021 
Tabla 3. Puntos de inflexión

\begin{tabular}{|c|c|c|c|}
\hline $\begin{array}{c}\text { Decreto } 1080 \text { de } 2015 \\
\text { Artículo 2.8.2.5.8. } \\
\text { Procesos de la gestión documental }\end{array}$ & NTC-ISO 30301 & NTC-ISO 27001 & NTC-ISO 9001 \\
\hline $\begin{array}{l}\text { Artículo 2.8.2.5.6. Política de gestión } \\
\text { documental. }\end{array}$ & 5.2 Política. & 5.2 Política. & 5.2 Política. \\
\hline Proceso de planeación: & $\begin{array}{l}\text { 4.1 Compresión de la organización. } \\
\text { 5.3.3. Responsabilidades } \\
\text { operacionales. } \\
\text { 6. Planificación. } \\
\text { 7.1 Recursos. } \\
\text { 7.3 Toma de conciencia. }\end{array}$ & $\begin{array}{c}\text { 4.1 Compresión de la organización. } \\
\text { 5.3 Roles, responsabilidades y } \\
\text { autoridades de la organización. } \\
\text { 6. Planificación. } \\
\text { 7.1 Recursos. } \\
\text { 7.3 Toma de conciencia. }\end{array}$ & $\begin{array}{c}\text { 4.2 Compresión de la organización. } \\
\text { 5.3 Roles, responsabilidades y } \\
\text { autoridades de la organización. } \\
\text { 6. Planificación. } \\
\text { 7.1 Recursos. } \\
\text { 7.3 Toma de conciencia. }\end{array}$ \\
\hline Proceso de producción: & $\begin{array}{c}\text { 7.5.1. Generalidades. } \\
\text { A.1.1.3 Crear reg. confiables. } \\
\text { 7.5.2 Control de la documentación. }\end{array}$ & $\begin{array}{l}\text { 7.5.1. Generalidades. } \\
\text { 7.5.2. Creación y actualización. } \\
\text { 7.5.3. Control de la información } \\
\text { documentada. }\end{array}$ & $\begin{array}{l}\text { 7.5.1. Generalidades. } \\
\text { 7.5.2. Creación y actualización. } \\
\text { 7.5.3.2 Control de la información. }\end{array}$ \\
\hline Proceso de gestión y trámite: & 7.5.2. Control de la documentación. & $\begin{array}{l}\text { 7.5.3. Control de la información } \\
\text { documentada. }\end{array}$ & $\begin{array}{l}\text { 7.5.3. Control de la información } \\
\text { documentada. }\end{array}$ \\
\hline Proceso de organización: & 7.5.2. Control de la documentación. & $\begin{array}{l}\text { 7.5.3. Control de la información } \\
\text { documentada. }\end{array}$ & $\begin{array}{l}\text { 7.5.3.2 Para el control de la } \\
\text { información documentada. }\end{array}$ \\
\hline Proceso de transferencias: & 7.5.2 Control de la documentación. & $\begin{array}{l}\text { 7.5.3. Control de la información } \\
\text { documentada. }\end{array}$ & $\begin{array}{l}\text { 7.5.3.1 La información documentada } \\
\text { requerida. }\end{array}$ \\
\hline proceso de disposición de documentos: & 7.5.2 Control de la documentación. & $\begin{array}{l}\text { 7.5.3. Control de la información } \\
\text { documentada. }\end{array}$ & $\begin{array}{l}\text { 7.5.3.2 Para el control de la } \\
\text { información documentada. }\end{array}$ \\
\hline Proceso de preservación: & 7.5.2 Control de la documentación. & $\begin{array}{l}\text { 7.5.3. Control de la información } \\
\text { documentada. }\end{array}$ & $\begin{array}{c}\text { 7.5.3.1 La información documentada } \\
\text { requerida. }\end{array}$ \\
\hline Proceso de valoración: & 7.5.2 Control de la documentación. & $\begin{array}{l}\text { 7.5.3. Control de la información } \\
\text { documentada. }\end{array}$ & $\begin{array}{l}\text { 7.5.3.2 Para el control de la } \\
\text { información documentada. }\end{array}$ \\
\hline
\end{tabular}

Fuente: elaboración propia (2019).

Signos, Investigación en Sistemas de Gestión

ISSN: 2145-1389 | e-ISSN: 2463-1140 | DOI: https://doi.org/10.15332/24631140

Vol. 13 N. ${ }^{\circ} 2$ | julio-diciembre de 2021 
En concordancia con las disposiciones normativas de los procesos de la gestión documental y los apartados de las NTC-ISO 30301, 27001 y 9001, se pudo establecer que los puntos de inflexión están inmersos principalmente en los aspectos referidos a la comprensión de la organización y su contexto, requisitos legales y otros, política, roles y responsabilidades, planificación, recursos, toma de conciencia e información documentada.

Con el diseño de la guía se logró desarrollar una propuesta metodológica de armonización basada en los puntos de inflexión y algunos elementos adicionales que sopesan las diferencias en cuanto a la estructura, contenidos y denominación de los procesos, aspectos, requisitos y en general que sirven para complementar las correlaciones de los sistemas de gestión documental, calidad y seguridad de la información. En la tabla 4 se presentan los resultados de la armonización propuesta.

Tabla 4. Metodología de armonización

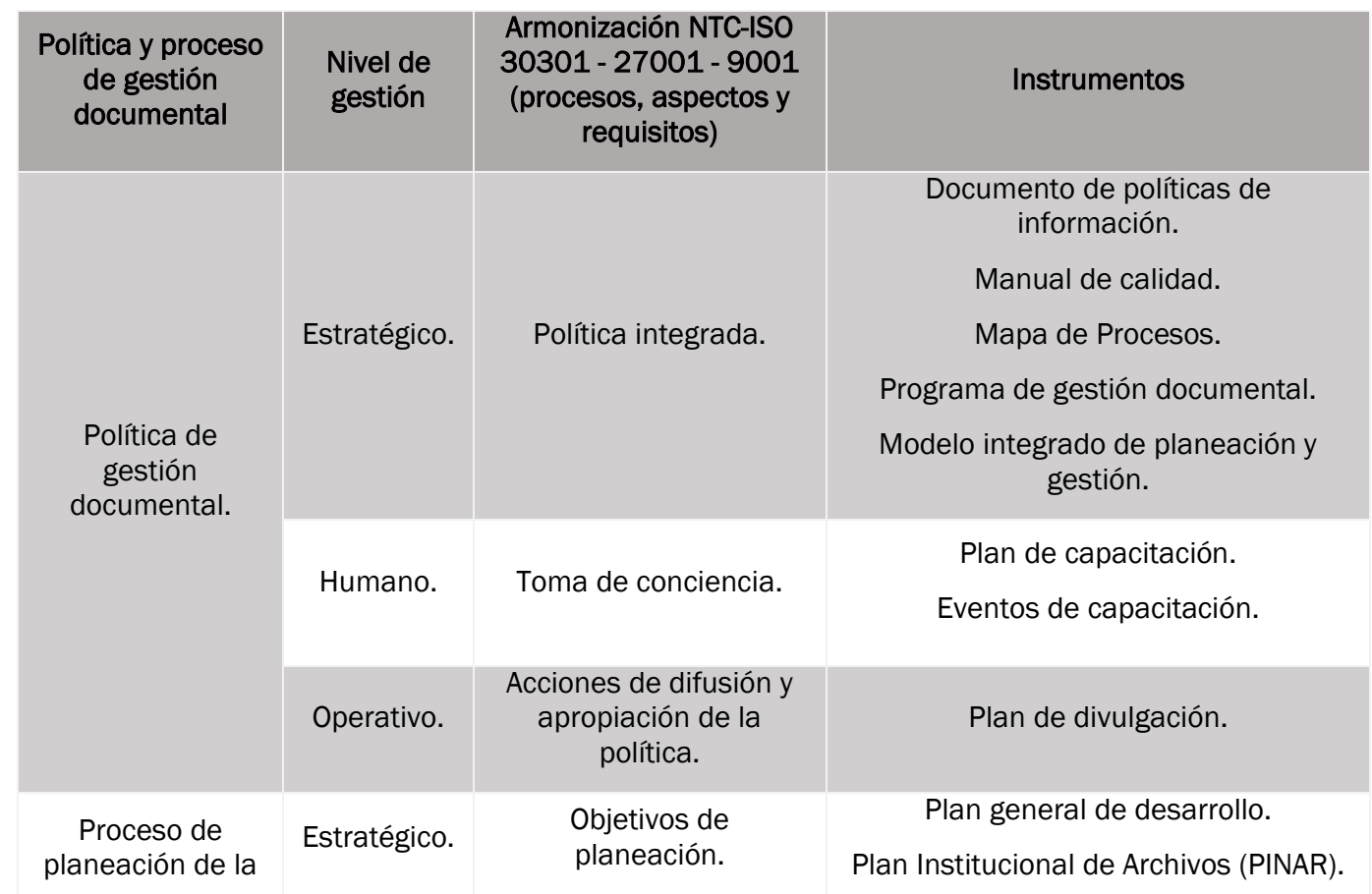

Signos, Investigación en Sistemas de Gestión

ISSN: 2145-1389 | e-ISSN: 2463-1140 | DOI: https://doi.org/10.15332/24631140

Vol. 13 N.o 2 | julio-diciembre de 2021 


\begin{tabular}{|c|c|c|c|}
\hline $\begin{array}{l}\text { Política y proceso } \\
\text { de gestión } \\
\text { documental }\end{array}$ & $\begin{array}{l}\text { Nivel de } \\
\text { gestión }\end{array}$ & $\begin{array}{c}\text { Armonización NTC-ISO } \\
30301 \text { - } 27001 \text { - } 9001 \\
\text { (procesos, aspectos y } \\
\text { requisitos) }\end{array}$ & Instrumentos \\
\hline \multirow[t]{3}{*}{$\begin{array}{c}\text { gestión } \\
\text { documental. }\end{array}$} & & $\begin{array}{c}\text { Comprensión de la } \\
\text { organización. } \\
\text { Determinación de } \\
\text { requisitos. } \\
\text { Identificación de riesgos } \\
\text { y oportunidades. } \\
\text { Mejora continua. } \\
\text { Evaluación y } \\
\text { seguimiento. } \\
\text { Lineamientos para la } \\
\text { planeación de la gestión } \\
\text { documental. } \\
\text { Lineamientos para la } \\
\text { seguridad de la } \\
\text { información. } \\
\text { Lineamientos para el } \\
\text { tratamiento de datos } \\
\text { personales. }\end{array}$ & $\begin{array}{c}\text { Plan de acción. } \\
\text { Matriz de riesgos. } \\
\text { Documento de políticas de } \\
\text { información. } \\
\text { Planes de mejora. } \\
\text { Indicadores. } \\
\text { Diagnóstico integral de archivos. } \\
\text { Sistema de Gestión de Documentos } \\
\text { Electrónicos de Archivo (SGDEA). } \\
\text { Programa de gestión documental. } \\
\text { Modelo integrado de planeación y } \\
\text { gestión. } \\
\text { Políticas de tratamiento de datos } \\
\text { personales. }\end{array}$ \\
\hline & Humano. & $\begin{array}{l}\text { Definición de roles y } \\
\text { responsabilidades. } \\
\text { Toma de conciencia. }\end{array}$ & $\begin{array}{l}\text { Manual de funciones. } \\
\text { Plan de capacitación. }\end{array}$ \\
\hline & Operativo. & $\begin{array}{l}\text { Actividades de } \\
\text { planificación de gestión } \\
\text { documental. } \\
\text { Determinación de } \\
\text { recursos. }\end{array}$ & $\begin{array}{c}\text { Diagnóstico integral de archivos. } \\
\text { Programa de gestión documental. } \\
\text { Plan de acción. } \\
\text { Plan de inversión. } \\
\text { Manual de procedimientos. } \\
\text { Instrumentos de transparencia } \\
\text { (esquema de publicación, registro de } \\
\text { activos de información e índice de } \\
\text { información clasificada y reservada). } \\
\text { Protocolo de firma electrónica. } \\
\text { Esquema de metadatos. } \\
\text { Plan de tratamiento de riesgos. }\end{array}$ \\
\hline \multirow{2}{*}{$\begin{array}{l}\text { Proceso de } \\
\text { producción de } \\
\text { documentos. }\end{array}$} & Estratégico. & $\begin{array}{l}\text { Lineamientos para la } \\
\text { captura, creación y } \\
\text { generación de } \\
\text { documentos. }\end{array}$ & $\begin{array}{l}\text { Documento de políticas de } \\
\text { información. } \\
\text { Programa de gestión documental. }\end{array}$ \\
\hline & Humano. & $\begin{array}{l}\text { Definición de roles y } \\
\text { responsabilidades. }\end{array}$ & Manual de funciones. \\
\hline
\end{tabular}

Signos, Investigación en Sistemas de Gestión

ISSN: 2145-1389 | e-ISSN: 2463-1140 | DOI: https://doi.org/10.15332/24631140

Vol. 13 N.O 2 | julio-diciembre de 2021 


\begin{tabular}{|c|c|c|c|}
\hline $\begin{array}{l}\text { Política y proceso } \\
\text { de gestión } \\
\text { documental }\end{array}$ & $\begin{array}{l}\text { Nivel de } \\
\text { gestión }\end{array}$ & $\begin{array}{c}\text { Armonización NTC-ISO } \\
30301 \text { - } 27001-9001 \\
\text { (procesos, aspectos y } \\
\text { requisitos) }\end{array}$ & Instrumentos \\
\hline & Operativo & $\begin{array}{l}\text { Definición de formatos y } \\
\text { documentos. } \\
\text { Criterios para la } \\
\text { creación y generación } \\
\text { de documentos. } \\
\text { Controles de versiones } \\
\text { de los documentos. }\end{array}$ & $\begin{array}{l}\text { Programa de gestión documental. } \\
\text { Manual de formas, formatos y } \\
\text { formularios. } \\
\text { Manual de procedimientos. } \\
\text { Manual de imagen corporativa. } \\
\text { Tablas de retención documental. } \\
\text { Manual de procedimientos. } \\
\text { Aplicativo de gestión documental. }\end{array}$ \\
\hline & Estratégico. & $\begin{array}{l}\text { Lineamientos para la } \\
\text { gestión y trámite. }\end{array}$ & $\begin{array}{l}\text { Documento de políticas de } \\
\text { información. } \\
\text { Programa de gestión documental. }\end{array}$ \\
\hline & Humano. & $\begin{array}{l}\text { Definición de roles y } \\
\text { responsabilidades. } \\
\text { Toma de conciencia. }\end{array}$ & $\begin{array}{l}\text { Manual de funciones. } \\
\text { Plan de capacitación. }\end{array}$ \\
\hline $\begin{array}{l}\text { Proceso de } \\
\text { gestión y trámite. }\end{array}$ & Operativo. & $\begin{array}{c}\text { Criterios para la } \\
\text { administración de la } \\
\text { correspondencia. } \\
\text { Controles para la } \\
\text { trazabilidad. } \\
\text { Controles criptográficos } \\
\text { y asignación de perfiles } \\
\text { de usuarios. } \\
\text { Directrices para } \\
\text { préstamo el préstamo } \\
\text { de documentos. } \\
\text { Definición de servicios } \\
\text { archivísticos. }\end{array}$ & $\begin{array}{l}\text { Programa de gestión documental. } \\
\text { Procedimiento de correspondencia. } \\
\text { Reglamento de préstamo. } \\
\text { Portafolio de servicios. } \\
\text { Aplicativo de gestión documental. } \\
\text { Tablas de control de acceso. }\end{array}$ \\
\hline \multirow{3}{*}{$\begin{array}{l}\text { Proceso de } \\
\text { organización. }\end{array}$} & Estratégico. & $\begin{array}{l}\text { Lineamientos para la } \\
\text { organización de los } \\
\text { documentos. }\end{array}$ & $\begin{array}{l}\text { Documento de políticas de } \\
\text { información. } \\
\text { Programa de gestión documental. }\end{array}$ \\
\hline & Humano. & $\begin{array}{l}\text { Definición de roles y } \\
\text { responsabilidades. } \\
\text { Toma de conciencia. }\end{array}$ & $\begin{array}{l}\text { Manual de funciones. } \\
\text { Plan de capacitación. }\end{array}$ \\
\hline & Operativo. & $\begin{array}{l}\text { Criterios para la } \\
\text { clasificación, } \\
\text { ordenación y } \\
\text { descripción de los } \\
\text { documentos. }\end{array}$ & $\begin{array}{l}\text { Programa de gestión documental. } \\
\text { Inventarios documentales. } \\
\text { Cuadro de clasificación. } \\
\text { Tablas de retención y valoración } \\
\text { documental. } \\
\text { Aplicativo de gestión documental. }\end{array}$ \\
\hline
\end{tabular}

Signos, Investigación en Sistemas de Gestión

ISSN: 2145-1389 | e-ISSN: 2463-1140 | DOI: https://doi.org/10.15332/24631140

Vol. 13 N.0 2 | julio-diciembre de 2021 


\begin{tabular}{|c|c|c|c|}
\hline $\begin{array}{l}\text { Política y proceso } \\
\text { de gestión } \\
\text { documental }\end{array}$ & $\begin{array}{l}\text { Nivel de } \\
\text { gestión }\end{array}$ & $\begin{array}{c}\text { Armonización NTC-ISO } \\
30301-27001-9001 \\
\text { (procesos, aspectos y } \\
\text { requisitos) }\end{array}$ & Instrumentos \\
\hline & & & Programa de descripción. \\
\hline \multirow{3}{*}{$\begin{array}{l}\text { Proceso de } \\
\text { transferencias. }\end{array}$} & Estratégico. & $\begin{array}{l}\text { Lineamientos para las } \\
\text { transferencias } \\
\text { documentales. }\end{array}$ & $\begin{array}{l}\text { Documento de políticas de } \\
\text { información. } \\
\text { Programa de gestión documental. }\end{array}$ \\
\hline & Humano. & $\begin{array}{l}\text { Definición de roles y } \\
\text { responsabilidades. } \\
\text { Toma de conciencia. }\end{array}$ & $\begin{array}{l}\text { Manual de funciones. } \\
\text { Plan de capacitación. }\end{array}$ \\
\hline & Operativo. & $\begin{array}{l}\text { Criterios para las } \\
\text { transferencias } \\
\text { primarias y } \\
\text { secundarias. }\end{array}$ & $\begin{array}{c}\text { Programa de gestión documental. } \\
\text { Aplicativo de gestión documental. } \\
\text { Inventarios documentales. } \\
\text { Procedimiento para las transferencias. } \\
\text { Procedimiento para la migración y } \\
\text { copias de seguridad. }\end{array}$ \\
\hline \multirow{3}{*}{$\begin{array}{l}\text { Proceso de } \\
\text { disposición de } \\
\text { documentos. }\end{array}$} & Estratégico. & $\begin{array}{l}\text { Lineamientos para la } \\
\text { disposición final de los } \\
\text { documentos. }\end{array}$ & $\begin{array}{l}\text { Documento de políticas de } \\
\text { información. } \\
\text { Programa de gestión documental. }\end{array}$ \\
\hline & Humano. & $\begin{array}{l}\text { Definición de roles y } \\
\text { responsabilidades. } \\
\text { Toma de conciencia. }\end{array}$ & $\begin{array}{l}\text { Manual de funciones. } \\
\text { Plan de capacitación. }\end{array}$ \\
\hline & Operativo. & $\begin{array}{l}\text { Criterios y actividades } \\
\text { para la conservación } \\
\text { permanente, selección, } \\
\text { digitalización y } \\
\text { eliminación de } \\
\text { documentos. }\end{array}$ & $\begin{array}{l}\text { Programa de gestión documental. } \\
\text { Tablas de retención y valoración } \\
\text { documental. } \\
\text { Aplicativo de gestión documental. } \\
\text { Procedimiento de digitalización. } \\
\text { Procedimiento de eliminación. }\end{array}$ \\
\hline \multirow{3}{*}{$\begin{array}{l}\text { Proceso de } \\
\text { preservación. }\end{array}$} & Estratégico. & $\begin{array}{l}\text { Lineamientos para la } \\
\text { preservación de la } \\
\text { información. }\end{array}$ & $\begin{array}{l}\text { Documento de políticas de } \\
\text { información. } \\
\text { Programa de gestión documental. } \\
\text { Sistema Integrado de Conservación } \\
\text { (SIC). }\end{array}$ \\
\hline & Humano. & $\begin{array}{l}\text { Definición de roles y } \\
\text { responsabilidades. } \\
\text { Toma de conciencia. }\end{array}$ & $\begin{array}{l}\text { Manual de funciones. } \\
\text { Plan de capacitación. }\end{array}$ \\
\hline & Operativo. & $\begin{array}{l}\text { Criterios y actividades } \\
\text { para la conservación, } \\
\text { preservación y } \\
\text { seguridad de la } \\
\text { información, los }\end{array}$ & $\begin{array}{l}\text { Programa de gestión documental. } \\
\text { Sistema Integrado de Conservación } \\
\text { (SIC). } \\
\text { Programa de documentos vitales. }\end{array}$ \\
\hline
\end{tabular}

Signos, Investigación en Sistemas de Gestión

ISSN: 2145-1389 | e-ISSN: 2463-1140 | DOI: https://doi.org/10.15332/24631140

Vol. 13 N.O 2 | julio-diciembre de 2021 


\begin{tabular}{|c|c|c|c|}
\hline $\begin{array}{l}\text { Política y proceso } \\
\text { de gestión } \\
\text { documental }\end{array}$ & $\begin{array}{l}\text { Nivel de } \\
\text { gestión }\end{array}$ & $\begin{array}{c}\text { Armonización NTC-ISO } \\
30301 \text { - } 27001 \text { - } 9001 \\
\text { (procesos, aspectos y } \\
\text { requisitos) }\end{array}$ & Instrumentos \\
\hline & & $\begin{array}{l}\text { sistemas, las redes, el } \\
\text { software y hardware. }\end{array}$ & $\begin{array}{l}\text { Aplicativo de gestión documental. } \\
\text { Inventario de activos de información. } \\
\text { Programa de reprografía. } \\
\text { Procedimiento para la migración. } \\
\text { Procedimiento para la seguridad de la } \\
\text { información. } \\
\text { Plan de contingencia y continuidad del } \\
\text { negocio. } \\
\text { Acuerdos de Confidencialidad. }\end{array}$ \\
\hline \multirow{3}{*}{$\begin{array}{l}\text { Proceso de } \\
\text { valoración. }\end{array}$} & Estratégico. & $\begin{array}{l}\text { Lineamientos para la } \\
\text { valoración de los } \\
\text { documentos. }\end{array}$ & $\begin{array}{l}\text { Documento de políticas de } \\
\text { información. } \\
\text { Programa de gestión documental. }\end{array}$ \\
\hline & Humano. & $\begin{array}{l}\text { Definición de roles y } \\
\text { responsabilidades. } \\
\text { Toma de conciencia. }\end{array}$ & $\begin{array}{l}\text { Manual de funciones. } \\
\text { Plan de capacitación. }\end{array}$ \\
\hline & Operativo. & $\begin{array}{l}\text { Criterios y actividades } \\
\text { para determinar los } \\
\text { valores primarios y } \\
\text { secundarios de los } \\
\text { documentos. }\end{array}$ & $\begin{array}{c}\text { Programa de gestión documental. } \\
\text { Tablas de retención y valoración } \\
\text { documental. }\end{array}$ \\
\hline
\end{tabular}

Fuente: elaboración propia (2020).

La aplicación del cuestionario de validación conformado por 14 preguntas, distribuidas así: de la 1 a la 4, asociadas a la claridad de la guía; de la 5 a la 9, asociadas a la pertinencia; y de la 10 a la 14, a la aplicabilidad.

La acotación de las respuestas es bajo los rangos: 1 = está totalmente en desacuerdo, 2 = está en desacuerdo, 3 = está de acuerdo, pero considera que se requiere ajustes, $4=$ está de acuerdo y $5=$ está totalmente de acuerdo. Esto permitió corroborar que entre los 3 expertos existió la aceptación de los criterios de los 14 ítems asociados a la claridad, pertenencia y aplicabilidad de la guía, de conformidad con los resultados obtenidos en el Coeficiente de concordancia W de Kendall (ver tabla 5): 
Tabla 5. Resultados de coeficiente de concordancia

\begin{tabular}{|c|c|c|c|}
\hline \multicolumn{4}{|c|}{ Coeficiente de concordancia W de Kendall } \\
\hline Datos / criterios & Claridad & Pertinencia & Aplicabilidad \\
\hline $\mathrm{T}$ & 54 & 72 & 73 \\
\hline $\mathrm{U}$ & 730 & 1038 & 1067 \\
\hline $\mathrm{n}$ & 4 & 5 & 5 \\
\hline $\mathrm{m}$ & 7 & 7 & 7 \\
\hline S & 1 & 1.2 & 1.2 \\
\hline W & 0.00487013 & 0.002735562 & 0.002757564 \\
\hline$X^{2}$ calculada & 0.102272727 & 0.076595745 & 0.077211796 \\
\hline $\mathbf{v}$ & 18 & 24 & 24 \\
\hline$\alpha \approx P$ & 0.05 & 0.05 & 0.05 \\
\hline $\mathrm{X}^{2}$ Tabla & 9.39 & 13.85 & 13.85 \\
\hline Aceptación Hipotesis & Ha: Aceptación criterio & Ha: Aceptación criterio & Ha: Aceptación criterio \\
\hline \multicolumn{4}{|c|}{ Coeficiente alfa de Cronbach } \\
\hline No. ítems & 14 & Alfa & 1.005432615 \\
\hline
\end{tabular}

Fuente: elaboración propia (2020).

Signos, Investigación en Sistemas de Gestión

ISSN: 2145-1389 | e-ISSN: 2463-1140 | DOI: https://doi.org/10.15332/24631140

Vol. 13 N.o 2 | julio-diciembre de 2021 
Teniendo en cuenta que el valor del coeficiente puede variar de o a 1 y que entre más se acerque el índice al extremo 1 mejor será la fiabilidad, se pudo comprobar la total aceptación de los ítems que componen la guía. Los objetivos de esta investigación se centraron en tener un conocimiento de la situación actual de los sistemas de gestión documental, calidad y seguridad de la información en una institución de educación superior para identificar los puntos de inflexión que permitieran diseñar una metodología de armonización entre dichos sistemas. Sus resultados se pueden enmarcar en la línea de continuidad de otras investigaciones similares desarrolladas en la maestría de Calidad y Gestión Integral, encaminadas a la formulación de mecanismos e instrumentos para que una organización tenga una mirada holística de la administración de su información y sus documentos, que integre los aspectos estratégicos, técnicos y operacionales de sus sistemas de gestión, de tal forma que facilite su recuperación oportuna y confiable para tomar decisiones. En este sentido, una organización puede tomar como punto de referencia cualquiera de estas investigaciones, de acuerdo con el grado de madurez alcanzado en la adopción de los sistemas de gestión referidos y de esta manera lograr su integración.

\section{Conclusiones}

A partir de los antecedentes de la investigación, se puedo evidenciar que las propuestas de integración de la gestión documental con los demás sistemas de gestión han estado orientadas a desarrollar instrumentos que faciliten su cohesión, tales como matrices para incorporar procesos y requisitos de los sistemas de gestión de calidad articulados con la Ley general de archivos. Así mismo, a formular estrategias orientadas a la identificación y definición de criterios para plantear la integración a partir de la planeación estratégica, la participación activa, el seguimiento y 
medición y el grado de alienación con los objetivos de una organización. Esto ha contribuido al reconocimiento de la gestión documental como un proceso muy importante para lograr el manejo integral y articulado de la información como un factor clave para el desarrollo organizacional.

Durante el análisis del contexto de la investigación, se observó que, a pesar de existir un gran avance y desarrollo normativo y metodológico sobre la gestión documental, hace falta una mayor claridad de los conceptos básicos de los procesos de la gestión documental para poder entender cómo deben estar en armonía con los demás sistemas de gestión de una organización y no solamente asociarse a los preceptos y principios generales de la Ley general de archivos.

La aplicación del diagnóstico sirvió para establecer que existe un alto cumplimiento de los procesos, actividades y requisitos planteados en la legislación archivística y las normas técnicas de gestión de calidad y seguridad de la información, sobre todo en lo atinente al compromiso de la alta dirección, formulación de políticas, toma de conciencia frente a la importancia de los sistemas, cumplimiento de requisitos e identificación de responsabilidades. Así mismo, las oportunidades de mejora en cada norma confluyen en los mismos aspectos, es decir que, para lograr la totalidad del cumplimiento, se requiere fortalecer la adquisición, desarrollo, uso y apropiación de herramientas, procedimientos y aplicaciones tecnológicas para la adecuada gestión de la calidad, documental y de seguridad de la información.

Por otro lado, la percepción que tienen las áreas que participaron en el diagnóstico frente al nivel de cumplimiento de los sistemas de gestión de la calidad, documental y seguridad de la información, es que se requiere mayor armonización de las actividades, porque se encuentran dispersas y 
pareciera que cada quien desarrolla actividades en forma aislada, lo cual impide contar con información confiable.

En cuanto a la identificación de los puntos de inflexión entre los procesos de gestión documental y los sistemas de gestión de calidad y seguridad de la información, se pudo establecer que están inmersos principalmente en los aspectos referidos a la comprensión de la organización y su contexto, requisitos legales, política, roles y responsabilidades, planificación, recursos, toma de conciencia e información documentada. Además, se puede lograr su armonización en pro de asegurar la adecuada administración, uso, manejo, seguridad, disposición y preservación de la información en una IES y dar respuesta a las necesidades de la comunidad universitaria.

Con el diseño y construcción de la "Guía para la armonización de los sistemas de gestión documental, calidad y seguridad de la información en una IES” se obtuvo una metodología que orienta las acciones para lograr la armonización de los procesos de la gestión documental en los niveles de gestión estratégico, humano y operativo; los puntos de inflexión entre las normas técnicas colombianas NTC-ISO 30301, 9001 y 27001; así como los documentos e instrumentos que orientan las políticas, procedimientos y actividades propios de una organización.

Como resultado del proceso de validación de la guía se logró confirmar la claridad y comprensión de los contenidos, la pertinencia, relevancia y aplicabilidad para su puesta en práctica. En tal sentido se destaca la percepción de los expertos como una herramienta que facilita entender la importancia de articular los diferentes sistemas de una organización; así mismo, es muy necesario para evidenciar cómo se da esa armonización y cómo las correlaciones de las diferentes actividades favorecen el control y seguimiento de las mismas. 
Finalmente, la armonización entre los sistemas de gestión de la calidad, documental y seguridad de la información propende por una mayor eficacia, eficiencia y oportunidad en el manejo de los documentos, en términos de asegurar la disponibilidad y aporte para la toma de decisiones, búsqueda de la mejora continua e impulso de la gestión del conocimiento e innovación como factores de desarrollo de una organización.

\section{Referencias}

Aja, L. (2002). Gestión de información, gestión del conocimiento y gestión de la calidad en las organizaciones. Acimed, $10(5), 2-6$.

http://scielo.sld.cu/scielo.php?script=sci arttext\&pid=S102494352002000500004

Archivo General de la Nación (2014). Guía para la implementación de un programa de gestión documental. AGN.

Archivo General de la Nación (2018). Modelo de Gestión Documental y Administración de Archivos. AGN.

Ayala, M. y Moreno, J. (2018). Guía para implementar la gestión documental armonizada con la gestión de la calidad y la Ley General de Archivo [tesis de maestría]. Universidad Santo Tomás.

Betancourt, A. y Caviedes, I. (2017). Propuesta metodológica de un Sistema Integrado de Gestión de Calidad en el Sector Salud articulado en el SUA con la NTC-ISO 90o1:2015 [tesis de maestría]. Universidad Santo Tomás.

Bustelo, C. (2012). Gestión de documentos en el contexto de Sistemas de Gestión ISO. Universitat Oberta.

Bustelo, C. (2015). La serie de normas ISO 30300 - management system for records: la gestión de los documentos integrada en la gestión de las organizaciones. Acervo, 28(2), $72-83$.

Casadesús, A. (2017). La normalización de la gestión documental más allá de los clásicos. Consultor de los ayuntamientos y de los juzgados, 7, 1-13.

Signos, Investigación en Sistemas de Gestión

ISSN: 2145-1389 | e-ISSN: 2463-1140 | DOI: https://doi.org/10.15332/24631140

Vol. 13 N. ${ }^{\circ} 2$ | julio-diciembre de 2021 
Castillo, J. y Osorio, C. (2011). La información documental para la implementación de sistemas de gestión de calidad aplicando la metodología de sistemas blandos. Anales de documentación, 14(1). https://dialnet.unirioja.es/servlet/articulo?codigo $=3946686$

Cipagauta, S. y Pachón, V. (2017). Definición de una guía metodológica para la implementación de un Programa de Gestión Documental en las pequeñas empresas de naturaleza privada, sustentada en la Resolución 8934 de 2014, emitida por Superintendencia de Industria y Comercio [tesis maestría]. Universidad de la Salle.

Decreto 1080 de 2015, por medio del cual se expide el decreto único reglamentario del sector cultura. Diario Oficial 49523.

http://www.suin-juriscol.gov.co/viewDocument.asp?ruta=Decretos/30019898

Departamento de Justicia País Vasco. (2011). Modelo de Gestión Documental.

Departamento de Justicia.

https://www.euskadi.eus/contenidos/informacion/modelo gestion documental/e u modgesdo/adjuntos/MGD 2.0.pdf

Escobar-Pérez, J., y Cuervo-Martínez, A. (2008). Validez de contenido y juicio de expertos: una aproximación a su utilización. Avances en Medición, 6, 27-36. https://www.researchgate.net/publication/302438451 Validez de contenido y juicio de expertos Una aproximacion_a su utilizacion

Hernández, K. (2011). Sistema de gestión de calidad en instituciones públicas de educación superior: estudio comparativo Universidad Nacional de ColombiaUniversidad del Valle [tesis maestría]. Universidad Nacional de Colombia.

Hernández, R. (2014). Metodología de la investigación. McGraw-Hill.

Instituto Colombiano de Normas Técnicas y Certificación [Icontec]. (2013). NTC-ISO 30301. Sistema de gestión para los documentos. Requisitos. Icontec.

Instituto Colombiano de Normas Técnicas y Certificación [Icontec]. (2013). NTCISO/IEC 27001. Tecnología de Información. Técnicas de Seguridad. Sistemas de Gestión de la Seguridad de la Información. Requisitos. Icontec.

Instituto Colombiano de Normas Técnicas y Certificación [Icontec]. (2015). NTC-ISO 90oo. Sistemas de gestión de la calidad fundamentos y vocabulario. Icontec. 
Instituto Colombiano de Normas Técnicas y Certificación [Icontec]. (2015). NTC-ISO 9001. Sistemas de gestión de la calidad. Requisitos. Icontec.

Instituto Colombiano de Normas Técnicas y Certificación [Icontec]. (2017). NTCISO/IEC 270oo. Tecnología de información. Técnicas de seguridad. Sistemas de gestión de seguridad de la información. Visión general y vocabulario. Icontec.

Integra. (2019). Sistemas de gestión. Integra. https://www.consultoresdesistemasdegestion.es/sistemas-de-gestion/

Llansó, J. (1993). Gestión de documentos: definición y análisis de modelos. http://eahahe.org/pdf/ikerlanak7.pdf

Ley 594 del 2000 (14 de junio), por medio de la cual se dicta la Ley general de archivos y se dictan otras disposiciones. Diario oficial 44084.

https://normativa.archivogeneral.gov.co/ley-594-de-2000/?pdf $=41$

Moreno, I. (2018). Estrategias para la integración de sistemas de gestión de calidad y sistemas de gestión documental, en una institución de educación superior. Signos, Investigación en Sistemas de Gestión, 1O(1), 113-125. https://www.redalyc.org/jatsRepo/5604/560459732006/html/index.html

Peña Guarín, G., Álvarez Álvarez, M. J. y Castro Rojas, M. (2013). Gestión del conocimiento: una perspectiva desde la gestión de la calidad y la gestión documental. Signos, Investigación en Sistemas de Gestión, 5(2), 101-118. https://doi.org/10.15332/s2145-1389.2013.0002.07

Peña Guarín, G. y Tejada, F. (2009). Reflexiones sobre las características constitutivas de la gestión integral. Signos, Investigación en Sistemas de Gestión, 1(2), 79-93. https://doi.org/10.15332/s2145-1389.2009.0002.08

Puentes, M. (2016) Propuesta metodológica para articular la gestión documental con los requisitos de la Ley General de Archivos y la Norma Técnica Internacional ISO 90o1:2015 [tesis de maestría]. Universidad Santo Tomás.

Rodríguez, B. (2002). El documento: entre la tradición y la renovación. Ediciones Trea.

Signos, Investigación en Sistemas de Gestión

ISSN: 2145-1389 | e-ISSN: 2463-1140 | DOI: https://doi.org/10.15332/24631140

Vol. 13 N.० 2 | julio-diciembre de 2021 